\title{
Recent Development of Bioenergy and Biorefinery in China
}

\author{
Prof. Mo Xian* (Assistant Director, Qingdao Institute of Bioenergy and Bioprocess \\ Technology, Chinese Academy of Sciences, China) \\ No. 163 Songling Road, Qingdao 266101, China
}

Received September 6, 2015; Published September 10, 2015

Although energy was used throughout the history of human culture, the burst of energy production and consumption coincided with the beginnings of the Industrial Revolution. The transition from hand production methods to machines, as well as the increasing exploration and use of fossil-derived fuels (coal, petroleum, natural gas, and shale fuel) made it possible to produce energy in large quantities. The majority of global energy supply still relies on traditional fossil based energy, with abundant fossil feedstock, well-understood technologies, and significant advantages in economy and scale. However, the traditional energy industry is now being challenged by sustainability of feedstock supply and environmental pressures, especially for carbon emission issues. Reducing the use of fossil fuels and replacing them with renewable energies have been widely accepted by scientists, businesses, and governors. Up to date, alternative renewable energies can be industrially produced via an extensive range of processes and sources like solar, wind, hydro, nuclear, and bioenergy. According to the data of World Bioenergy Association (www.worldbioenergy.org), bioenergy has now been the largest global renewable energy supply, which is only lower than the traditional fossil energy.

China has abundant biomass resources that are about 0.4 billion tons per year - if totally utilized for generating bioenergy, it can satisfy $10 \%$ of total energy consumption in China. However, the current utilization of bioenergy in China is of low efficiency due to immature technologies, small-scale productions, and limited market channels. For example, in a global level, the final energy from biomass is bioheat, followed by transport fuels (mainly as corn/cellulose ethanol, biodiesel) and electricity. While in China, bioelectricity is the main form of bioenergy, large quantities of biomass are not efficiently utilized. Straw burning is still widely present in rural areas during the harvesting season, which not only wastes the valuable bioresources but also causes severe air pollution.

Currently, national projects are prompted in research and pilot levels to improve the quantity and quality of Chinese bioenergy framework. Integrated production processes for both bioheat and electricity were developed in recent years which aimed to improve the conversion efficiency of bioenergy. Super biogas projects are prompted in many regions where have abundant biomass resources. Second generation bio-ethanol achieved pilot-scale production, which is utilizing non-food agriculture wastes like the corncobs. Moreover, biorefinery and bioconversion of the biomass to value added products are in early stages of development. Exploration of advanced bio-fuels and valueadded chemical building blocks using modern techniques are future trends in the business of bioenergy and biorefinery. A variety of bio-, chemo- processes may be integrated in 
conversion of the biomass to complex products. For example, Sinopec, one of the Chinese leading chemical companies, has explored 1st generation of bio-jet fuel, which is made from waste cooking oil via a variety of chemical processing techniques. Similar projects are in fast developing by industries as well as the research institutes.

It is hard to answer what is the future of bioenergy and biorefinery in China. As nobody in the last century can predict the scale and the diversity of the relative business of bioenergy in nowadays. But this industry will have a promising future: a seemingly infinite and renewable bio- resources in our lands, huge members of industries, governments and research institutes are eager to contribute their endeavors in this fast developing field, and more discoveries and modern techniques will be unfolded in the near future. We will walk a long way to fill in the gap between pioneering projects and conceptions and the practical scale-up application in this field.

Article copyright: (C) 2015 Mo Xian. This is an open access article distributed under the terms of the Creative Commons Attribution 4.0 International License, which permits unrestricted use and distribution provided the original author and source are credited.

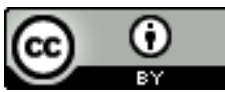

